\title{
Treatment-Dose LMWH versus Prophylactic/ Intermediate Dose Heparins in High-Risk COVID-19 Inpatients: Rationale and Design of the HEP-COVID Trial
}

\author{
Mark Goldin ${ }^{1,2}$ Dimitrios Giannis ${ }^{1}$ Wassim Diab ${ }^{2}$ Janice Wang ${ }^{1,2}$ Sameer Khanijo ${ }^{2}$ \\ Gulru Sharifova ${ }^{2}$ Marc Cohen ${ }^{3}$ Jeet M. Lund ${ }^{4}$ Andrea Mignatti ${ }^{2,5}$ Eugenia Gianos 2,5 \\ Alfonso Tafur ${ }^{6,7}$ Paul A. Lewis ${ }^{8}$ Kevin Cohoon ${ }^{9}$ John M. Kittelson ${ }^{10}$ Martin L. Lesser ${ }^{1,2}$ \\ Cristina P. Sison ${ }^{1,2}$ Husneara Rahman ${ }^{1}$ Kanta Ochani ${ }^{1}$ William R. Hiatt ${ }^{\dagger 11,12}$ Rita A. Dale ${ }^{11}$ \\ Victoria E. Anderson ${ }^{11}$ Marc Bonaca ${ }^{11,12}$ Jonathan L. Halperin ${ }^{13}$ Jeffrey I. Weitz ${ }^{14-16}$
} Alex C. Spyropoulos ${ }^{1,2}$

${ }^{1}$ Institute of Health Innovations and Outcomes Research, The Feinstein Institutes for Medical Research, Northwell Health, Manhasset, New York, United States

2 Department of Medicine, Donald and Barbara Zucker School of Medicine at Hofstra/Northwell, Hempstead, New York, United States

${ }^{3}$ Department of Medicine, Newark Beth Israel Medical Center, Newark, New Jersey, United States

${ }^{4}$ Wellspan York Hospital, York, Pennsylvania, United States

${ }^{5}$ Department of Cardiology, Lenox Hill Hospital, Northwell Health, New York, New York, United States

${ }^{6}$ Department of Medicine and Vascular Medicine, NorthShore University Health System, Evanston, Illinois, United States

${ }^{7}$ Division of Cardiology, University of Chicago, Pritzker School of Medicine, Chicago, Illinois, United States

8 Evidence Based Medicine, Baycare Health System, Clearwater, Florida, United States

${ }^{9}$ Division of Cardiovascular Medicine, Department of Medicine, Froedtert and Medical College of Wisconsin, Milwaukee, Wisconsin, United States

Thromb Haemost 2021;121:1684-1695.
Address for correspondence Alex C. Spyropoulos, MD, FACP, FCCP, FRCPC, Anticoagulation and Clinical Thrombosis Services, Northwell Health at Lenox Hill Hospital, 130 East 77th Street, New York, NY 10075, United States (e-mail: aspyropoul@northwell.edu).

10 Department of Biostatistics and Informatics, Colorado School of Public Health, University of Colorado Anschutz Medical Campus, Aurora, Colorado, United States

11 Biostatistics, Colorado Prevention Center (CPC) Clinical Research, Aurora,Colorado, United States

12 Division of Cardiology, University of Colorado, School of Medicine, Aurora, Colorado, United States

13 Department of Cardiovascular Medicine, The Cardiovascular Institute, Mount Sinai Medical Center, New York, New York, United States

${ }^{14}$ Department of Medicine, McMaster University, Hamilton, Ontario, Canada

${ }^{15}$ Department of Biochemistry and Biomedical Sciences, McMaster University, Hamilton, Ontario, Canada

${ }^{16}$ Thrombosis and Atherosclerosis Research Institute, Hamilton, Ontario, Canada
Abstract
Keywords
- coronavirus disease- 2019
- venous thromboembolism
- arterial thromboembolism
- thromboprophylaxis
- D-dimer

Coronavirus disease-2019 (COVID-19) has been associated with significant risk of venous thromboembolism (VTE), arterial thromboembolism (ATE), and mortality particularly among hospitalized patients with critical illness and elevated D-dimer (Dd) levels. Conflicting data have yet to elucidate optimal thromboprophylaxis dosing. HEP-COVID (NCT04401293) is a phase 3 , multicenter, pragmatic, prospective, randomized, pseudo-blinded, active control trial to evaluate efficacy and safety of therapeutic-dose low-molecular-weight heparin (LMWH) versus prophylactic-/intermediate-dose LMWH or unfractionated heparin (UFH) for prevention of a primary efficacy composite outcome of VTE, ATE, and all-cause mortality $30 \pm 2$ days post-enrollment. Eligible patients have COVID-19 diagnosis by nasal swab or serologic testing, requirement for supplemental oxygen per investigator judgment, and Dd $>4 \times$ upper limit of normal (ULN) or sepsis-induced coagulopathy score $\geq 4$. Subjects are randomized to enoxaparin $1 \mathrm{mg} / \mathrm{kg}$ subcutaneous (SQ)/two times a day (BID) (creatinine

Deceased.

received

January 22, 2021

accepted

April 1, 2021

published online

April 6, 2021 (c) 2021. Thieme. All rights reserved. Georg Thieme Verlag KG,

Rüdigerstraße 14,

70469 Stuttgart, Germany
DOI https://doi.org/

10.1055/a-1475-2351.

ISSN 0340-6245. 
clearance $[\mathrm{CrCl}] \geq 30 \mathrm{~mL} / \mathrm{min})$ or $0.5 \mathrm{mg} / \mathrm{kg}(\mathrm{CrCl} 15-30 \mathrm{~mL} / \mathrm{min})$ versus local institutional prophylactic regimens including (1) UFH up to $22,500 \mathrm{IU}$ (international unit) daily (divided BID or three times a day), (2) enoxaparin 30 and $40 \mathrm{mg}$ SQ QD (once daily) or BID, or (3) dalteparin 2,500 IU or 5,000 IU QD. The principal safety outcome is major bleeding. Events are adjudicated locally. Based on expected $40 \%$ relative risk reduction with treatment-dose compared with prophylactic-dose prophylaxis, 308 subjects will be enrolled (assuming 20\% drop-out) to achieve $80 \%$ power. Distinguishing design features include an enriched population for the composite endpoint anchored on Dd $>4 \times$ ULN, stratification by intensive care unit (ICU) versus non-ICU, and the ability to capture asymptomatic proximal deep venous thrombosis via screening ultrasonography prior to discharge.

\section{Introduction}

The coronavirus disease-2019 (COVID-19) pandemic has led to nearly 80 million cases of acute viral illness and over 1.7 million deaths globally, and at the close of 2020, more than 500,000 new cases and 10,000 deaths were reported daily, both with steep upward trends. The United States accounts for nearly one-quarter of total cases and more than one-fifth of deaths, and currently over 118,000 individuals are hospitalized due to COVID-19. ${ }^{1}$

Although SARS-CoV-2 is primarily a respiratory virus, COVID-19 that is caused by the virus has myriad systemic manifestations, and thrombosis is a major cause of morbidity and mortality in hospitalized patients. Thrombotic events in such patients include venous thromboembolism (VTE), such as deep vein thrombosis (DVT) and pulmonary embolism (PE), and arterial thromboembolism (ATE), such as myocardial infarction (MI) and ischemic stroke. ${ }^{2-5}$ Proposed risk factors for and mechanisms of thrombosis include acute illness with patient-related underlying risk factors and associated immobility, as well as cytokine storm and direct viral effects on the endothelium with coagulation activation. ${ }^{6-9}$

Earlier studies reported VTE rates of $46 \%$ or greater in critically ill hospitalized patients with COVID-19, while more recent reports in larger U.S. studies suggest much lower rates of 1.7 to $3.6 \%$ in patients with COVID-19 admitted to medical wards. ${ }^{3,4,10}$ Alarmingly, autopsy data suggest that more than $60 \%$ of patients have undiagnosed DVT or in situ pulmonary microthrombi at the time of death. ${ }^{11,12}$ Additionally, there remain subgroups of hospitalized COVID-19 medical patients, especially those with critical illness, that experience "breakthrough thrombosis" despite standard thromboprophylaxis., 2,13 Furthermore, elevated plasma levels of D-dimer-in addition to other laboratory parameters such as C-reactive protein and interleukin-6-have been associated with increased thrombotic risk and poor outcomes in patients hospitalized with COVID-19. ${ }^{14}$ Thus, hospitalized COVID-19 patients with high levels of D-dimer represent a critical cohort for studying optimal thromboprophylaxis dosing.

Retrospective studies have suggested a possible role for empiric treatment-dose anticoagulant regimens for thromboprophylaxis, but there remains a paucity of high-quality clinical trial data to confirm any potential net clinical benefit of this strategy. ${ }^{15-17}$ Given the unique characteristics of COVID-19 coagulopathy, guidance statements on hospitalized COVID-19 patients promote universal anticoagulant thromboprophylaxis and identify escalated anticoagulant dosing as a strategy to consider in high-risk groups, including those with critical illness. ${ }^{7,18-20}$ However, all guidance statements have noted an urgent need for high-quality randomized trials to test the hypothesis that escalated or treatment-dose anticoagulant therapy provides a net clinical benefit over conventional low-dose anticoagulant regimens for thromboprophylaxis in hospitalized COVID-19 patients. ${ }^{7,18-20}$

This article describes the ongoing Systemic Anticoagulation with Full-Dose Low Molecular Weight Heparin (LMWH) versus Prophylactic or Intermediate-Dose LMWH/Unfractionated Heparin (UFH) in High-Risk COVID-19 Patients (HEP-COVID) trial including the (1) rationale and design, (2) main objectives, and (3) potential clinical implications.

\section{Methods}

\section{Study Objectives and Hypothesis}

The primary objective of the study is to evaluate the efficacy and safety of therapeutic-dose LMWH compared with prophylactic/intermediate doses of LMWH or UFH for the prevention of the primary composite efficacy endpoint of VTE, ATE, and all-cause mortality (ACM) at $30 \pm 2$ days after study enrollment. The key secondary objective includes testing the hypothesis that therapeutic-dose LMWH will be superior to prophylactic/intermediate doses of LMWH/UFH for the prevention of the composite of VTE, ATE, and ACM at day $10+4$ after hospital admission. Other secondary objectives include testing the hypothesis that therapeutic-dose LMWH will be superior to prophylactic/intermediate doses of LMWH/UFH for the prevention of progression to acute respiratory distress syndrome (ARDS), new-onset atrial fibrillation (AF), acute kidney injury (AKI), nonfatal cardiac arrest, need for intubation, and rehospitalization at day $30 \pm 2$.

The principal safety endpoint is major bleeding as defined by the International Society on Thrombosis and Haemostasis (ISTH) at $30 \pm 2$ days after enrollment. The primary and secondary efficacy, principal safety, and other key secondary outcomes are summarized in -Table $\mathbf{1}$. 
Table 1 Outcomes of interest in the HEP-COVID trial

\begin{tabular}{|c|c|}
\hline \multirow[t]{3}{*}{$\begin{array}{l}\text { Composite of ATE, VTE, and ACM } \\
\text { ( } 30 \pm 2 \text { days after enrollment) }\end{array}$} & $\begin{array}{l}\text { ATE: } \\
\text { - Documented thromboembolic stroke by imaging (head CT, brain MRI) defined as a new } \\
\text { focal neurologic defect lasting at least } 24 \text { hours that is not due to a readily identifiable } \\
\text { nonvascular cause } \\
\text { - Documented peripheral arterial thromboembolism by imaging (CT scan, arteriography, } \\
\text { arterial Doppler of extremities) } \\
\text { - Documented acute myocardial infarction defined by two of the three following } \\
\text { conditions: ( } 1 \text { ) an appropriate clinical condition such as new EKG changes; ( } 2 \text { ) elevation } \\
\text { of CK-MB or troponin-T or I } \geq 2 \times \text { ULN (if CK-MB or troponin unavailable then total } \\
\text { CK } \geq 2 \times \text { ULN); (3) new significant ( } \geq 0.04 \mathrm{~s}) \mathrm{Q} \text { waves in } \geq 2 \text { contiguous leads }\end{array}$ \\
\hline & $\begin{array}{l}\text { VTE: } \\
\text { Any new venous thromboembolic event (symptomatic DVT or asymptomatic proximal DVT } \\
\text { found by screening ultrasonography or as an incidental finding of PE on CT scan) including } \\
\text { DVT of upper or lower extremities, PE, splanchnic vein thrombosis, cerebral vein thrombosis } \\
\text { defined by: } \\
\text { - One or more new filing defects at compression ultrasonography, venography, CT } \\
\text { venography, or MR venography } \\
\text { - A new perfusion defect of at least } 75 \% \text { of a segment with a local normal ventilation result } \\
\text { (high probability) on ventilation/perfusion scan (V/Q scan) } \\
\text { - A noncompressible venous segment on compression ultrasonography, or in patients with } \\
\text { a history of previous DVT, either a new noncompressible segment or a substantial } \\
\text { increase (4 mm or more) in the diameter of the vein during full compression in a } \\
\text { previously abnormal segment on ultrasonography } \\
\text { - In the absence of an imaging test in a hemodynamically unstable patient, evidence of } \\
\text { right ventricular dysfunction by transthoracic or transesophageal echocardiogram } \\
\text { (ESC Criteria) }\end{array}$ \\
\hline & All-cause mortality \\
\hline \multicolumn{2}{|l|}{ Secondary efficacy outcome } \\
\hline $\begin{array}{l}\text { Composite of ATE, VTE, and ACM } \\
(10+4 \text { days after } \\
\text { hospital admission })\end{array}$ & Same as primary efficacy outcome + asymptomatic distal DVT of the lower extremity \\
\hline \multicolumn{2}{|l|}{ Principal safety outcome } \\
\hline Major bleeding & $\begin{array}{l}\text { Documented major bleeding using ISTH criteria defined by: } \\
\text { - A decrease in hemoglobin of } 2 \mathrm{~g} / \mathrm{dL} \text { or more within } 24 \text { hours } \\
\text { - A transfusion of } 2 \text { or more units of packed red blood cells } \\
\text { - Critical site bleeding (including intracranial, intraocular, intra-articular, retroperitoneal, } \\
\text { intramuscular with component syndrome, pericardial) } \\
\text { - Bleeding that is fatal } \\
\text { - Bleeding that necessitates surgical intervention }\end{array}$ \\
\hline \multicolumn{2}{|l|}{ Other secondary outcomes } \\
\hline \multicolumn{2}{|c|}{ Progression to acute respiratory distress syndrome (ARDS) } \\
\hline \multicolumn{2}{|l|}{ Rehospitalization } \\
\hline \multicolumn{2}{|l|}{ Need for intubation } \\
\hline \multicolumn{2}{|l|}{ New-onset atrial fibrillation } \\
\hline \multicolumn{2}{|l|}{ Nonfatal cardiac arrest } \\
\hline Acute kidney injury & \\
\hline
\end{tabular}

Abbreviations: ACM; all-cause mortality; ATE; arterial thromboembolism; CK-MB, creatine kinase-myocardial band; CT, computed tomography; DVT, deep vein thrombosis; EKG, electrocardiogram; ESC, European Society of Cardiology; ISTH, International Society on Thrombosis and Haemostasis; MRI, magnetic resonance imaging; PE, pulmonary embolism; ULN, upper limit of normal; VTE, venous thromboembolism.

\section{Study Overview}

HEP-COVID (NCT04401293) is a phase 3, multicenter, pragmatic, prospective, randomized pseudo-blinded, active control trial that is conducted both in hospitals within the Northwell Health system in New York and in other health care systems in the United States. Study enrollment began in
May 2020 and is expected to continue for approximately 1 year until our target patient enrollment is achieved.

The study consists of a (1) screening phase (within 72 hours after hospital admission), (2) pseudo-blinded treatment phase beginning at the time of enrollment and ending at hospital discharge or the occurrence of a primary or 


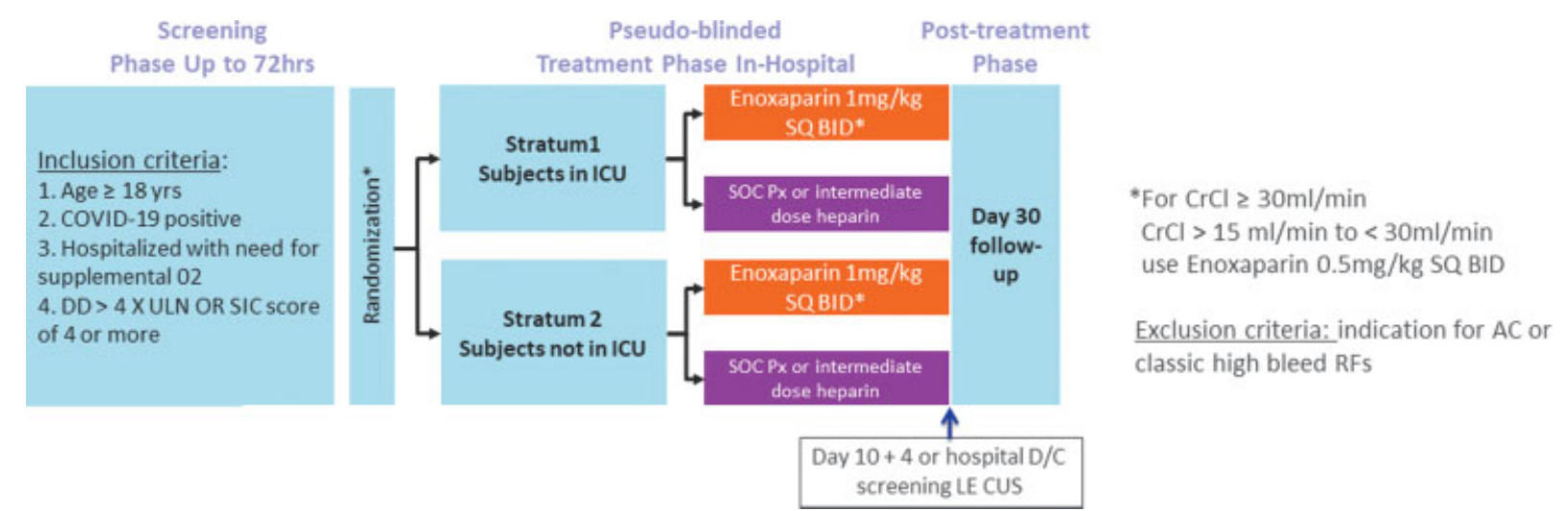

Fig. 1 The design of the HEP-COVID randomized pseudo-blinded active control trial (NCT04401293).

key secondary efficacy endpoint or principal safety endpoint requiring discontinuation of the study medication, with all patients receiving a lower extremity screening Doppler compression ultrasound (CUS) at hospital day $10+4$ or sooner at the time of discharge, and (3) a $30 \pm 2$ day follow-up period (-Fig. 1).

The study is coordinated by the study co-principal investigators (PIs), an executive committee (EC) composed of members of the academic leadership of the study, and site PIs. An independent data and safety monitoring board (DSMB) monitors patient safety and outcomes at predetermined intervals during the study and makes recommendations to the EC regarding ongoing trial conduct.

The trial protocol was reviewed by regulatory authorities, including Northwell Institutional Review Board (IRB) and external IRBs at each center. Informed consent is obtained from eligible patients or their designates prior to enrollment.

\section{Treatment Arms and Rationale}

The optimal dose of heparin (either LMWH or UFH) in hospitalized COVID-19 patients is unknown because there are reports that patients receiving conventional prophylactic dose heparin (UFH or LMWH) as supported by international guidance statements on hospitalized COVID-19 patients remain at risk for thromboembolic events. ${ }^{18}$ There are data to support improved efficacy with treatment doses of twice-daily enoxaparin versus once-daily (QD) weight-adjusted enoxaparin for the management of VTE, especially with large thrombus burden. ${ }^{21}$ There are also data to support the concept that treatment-dose heparin reduces major cardiovascular events. ${ }^{22}$ Our current standard of care in the 24-hospital Northwell Health System, which has hospitalized a large number of patients with COVID-19, is to use enoxaparin $40 \mathrm{mg}$ subcutaneous (SQ) QD for patients with a body mass index (BMI) $<30$ and creatinine clearance $(\mathrm{CrCl})>15 \mathrm{~mL} / \mathrm{min}$, enoxaparin $40 \mathrm{mg}$ SQ twice daily (BID) for patients with a BMI $>30$ and $\mathrm{CrCl}$ $>15 \mathrm{~mL} / \mathrm{min}$, and UFH 5,000 U SQ BID or three times a day (TID) in patients with a $\mathrm{CrCl}<15 \mathrm{~mL} / \mathrm{min}$ and $\mathrm{BMI}<30$ and UFH $7,500 \mathrm{U}$ SQ BID or TID with a $\mathrm{CrCl}<15 \mathrm{~mL} / \mathrm{min}$ and BMI $>30$. Large health care institutions in the United States and elsewhere have protocols for in-patient thromboprophylaxis ranging from prophylactic to intermediate-dose UFH or LMWH for the management of hospitalized patients with COVID-19associated coagulopathy. ${ }^{23}$

\section{Patient Population and Eligibility}

The patient population consists of adult ( $\geq 18$ years of age) males and nonpregnant females at the time of enrollment, with a positive COVID-19 diagnosis by nasal swab or serologic testing, who require supplemental oxygen as per investigator's judgment and either have a D-dimer level $\geq 4 \times$ the upper limit of the local laboratory normal (ULN) or a sepsis-induced coagulopathy (SIC) score $\geq 4$. The patient (or a legally authorized representative) must be able to provide written informed consent prior to enrollment, and must understand and agree to comply with planned study procedures. The patient must consent to randomization within 72 hours after hospital admission or within 72 hours of index hospitalization if the patient was transferred from another facility.

Patients with a medical condition that requires administration of any parenteral or oral anticoagulant during the screening phase are not eligible for participation. Patients with an absolute contraindication to anticoagulation, including active bleeding, recent (within 1 month) history of bleeding, dual antiplatelet therapy, active gastrointestinal or intracranial cancer, history of bronchiectasis or pulmonary cavitation, liver failure with baseline international normalized ratio higher than $1.5, \mathrm{CrCl}$ less than $15 \mathrm{~mL} / \mathrm{min}$, platelet count lower than 25,000 , history of heparin-induced thrombocytopenia within the past 100 days in the presence of circulating antibodies using standardized definitions, ${ }^{24}$ and hypersensitivity to enoxaparin/heparin/pork products/benzyl alcohol are not eligible for participation. In addition, patients participating in another blinded trial of investigational drug therapy for COVID-19 are excluded. The full list of inclusion and exclusion criteria is provided in $\mathbf{- T a b l e ~} \mathbf{2}$.

\section{Randomization and Stratification}

The Feinstein Institutes Biostatistics Unit (Northwell Health) developed and implemented the randomization procedure using the Biostatistics Randomization Management System (BRMS). BRMS is a secure, HIPAA-compliant, web-based application that allows investigators to randomize subjects into randomized clinical trials (RCTs) using their personal 
Table 2 Inclusion and exclusion criteria

\begin{tabular}{|l|l|}
\hline \multicolumn{2}{|l|}{ Inclusion criteria } \\
\hline 1 & $\begin{array}{l}\text { Subject (or legally authorized representative) } \\
\text { provides written informed consent prior to } \\
\text { initiation of any study procedures }\end{array}$ \\
\hline 2 & $\begin{array}{l}\text { Understands and agrees to comply with planned } \\
\text { study procedures }\end{array}$ \\
\hline 3 & $\begin{array}{l}\text { Male or nonpregnant female adult } \geq 18 \text { years of } \\
\text { age at the time of enrollment }\end{array}$ \\
\hline 4 & $\begin{array}{l}\text { Subjects with a positive COVID-19 diagnosis by } \\
\text { nasal swab or serologic testing }\end{array}$ \\
\hline 5 & $\begin{array}{l}\text { Subject consents to randomization within 72 hours } \\
\text { of hospital admission or transfer from another } \\
\text { facility within } 72 \text { hours of index presentation }\end{array}$ \\
\hline 6 & $\begin{array}{l}\text { Hospitalized with a requirement for supplemental } \\
\text { oxygen }\end{array}$ \\
\hline 7 & $\begin{array}{l}\text { D-Dimer }>4.0 \times \text { ULN OR sepsis-induced } \\
\text { coagulopathy (SIC) score of } \geq 4\end{array}$ \\
\hline Exclusion criteria \\
\hline 1 & $\begin{array}{l}\text { A medical condition that requires administration of } \\
\text { any parenteral or oral anticoagulant }\end{array}$ \\
\hline 2 & Active bleeding \\
\hline 3 & $\begin{array}{l}\text { Recent ( }<1 \text { month) history of bleeding } \\
\text { investigational drug therapy for COVID-19 }\end{array}$ \\
\hline 4 & Dual antiplatelet therapy \\
\hline 5 & $\begin{array}{l}\text { Active gastrointestinal or intracranial cancer } \\
\text { hypersensitivity to enoxaparin sodium, } \\
\text { hypersensitivity to benzyl alcohol }\end{array}$ \\
\hline 12 & $\begin{array}{l}\text { History of bronchiectasis or pulmonary cavitation } \\
\text { circulating antibodies }\end{array}$ \\
\hline 10 & CrCl $<15$ mL/min \\
\hline 14
\end{tabular}

Abbreviations: $\mathrm{CrCl}$, creatinine clearance; INR, international normalized ratio; PLT, platelet; ULN, upper limit of normal.

computer. BRMS allows for multicenter, stratified, and single/double-blinded RCTs, using permuted blocks.

Eligible patients will be stratified according to whether their level of care corresponds to intensive care unit (ICU) care or not. During the COVID-19 pandemic, a health system's ICUs may become overwhelmed so that critically ill patients with COVID-19 may be located outside a designated ICU. As such, the definitions of what constitutes a patient with ICU-level care at the time of randomization can include (1) subjects who require noninvasive ventilation acutely for their COVID-19- related respiratory issues (high flow nasal cannula, bi-level ventilation, average volume-assured pressure support, or continuous positive airway pressure, (2) subjects who require vasopressor therapy, and (3) subjects who require vital sign monitoring more often than every 4 hours ( $q 4 h$ ).

Subjects will be randomly assigned to the treatment arm (arm 0: treatment dose of LMWH) or the prophylactic-/ intermediate-dose arm (arm 1: prophylactic/intermediate dose of LMWH or UFH) in a 1:1 ratio. The treatment-dose arm consists of enoxaparin $1 \mathrm{mg} / \mathrm{kg}$ SQ/BID in patients with $\mathrm{CrCl}$ $\geq 30 \mathrm{~mL} / \mathrm{min}$ or enoxaparin $0.5 \mathrm{mg} / \mathrm{kg}$ in patients with $\mathrm{CrCl}$ $\geq 15 \mathrm{~mL} / \mathrm{min}$ and $<30 \mathrm{~mL} / \mathrm{min}$ at the time of randomization. The prophylactic-/intermediate-dose LMWH or UFH arm follows the local institutional standard of care and includes the following regimens: (1) UFH up to 22,500 IU daily in BID or TID doses (i.e., UFH $5,000 \mathrm{IU}$ SQ BID/TID or $7,500 \mathrm{IU}$ BID/TID), (2) enoxaparin 30 and $40 \mathrm{mg}$ SQ QD or BID (the use of weight-based enoxaparin, i.e., $0.5 \mathrm{mg} / \mathrm{kg}$ SQ BID for this arm is acceptable, but strongly discouraged), or (3) dalteparin 2,500 IU or 5,000 IU QD. The first dose of study drug is to be given as soon after randomization as possible and the treatment period will be for the duration of hospitalization or until a primary or key secondary efficacy or principal safety endpoint is met. If any of these study endpoints is met, the subject is taken off study drug and placed on open-label anticoagulant therapy.

Individual dose modification is not permitted unless the $\mathrm{CrCl}$ falls below $15 \mathrm{~mL} / \mathrm{min}$ in the treatment arm (arm 0 ). In that case, conversion to dose-adjusted intravenous (IV) UFH is acceptable. The investigator is encouraged to convert back to treatment-dose enoxaparin as per protocol once the $\mathrm{CrCl}$ returns to values higher than or equal to $15 \mathrm{~mL} / \mathrm{min}$. Dose modification is allowed in the prophylactic/intermediate group (arm 1) if the $\mathrm{CrCl}$ falls below $15 \mathrm{~mL} / \mathrm{min}$ so that UFH up to $22,500 \mathrm{U}$ daily (i.e., UFH $5,000 \mathrm{U}$ SQ BID or TID or 7,500 IU SQ BID or TID) can be used. The investigator is encouraged to convert back to prophylactic-/intermediatedose $\mathrm{LMWH} / \mathrm{UFH}$ as per protocol once the $\mathrm{CrCl}$ returns to values higher than or equal to $15 \mathrm{~mL} / \mathrm{min}$. If a subject requires permanent discontinuation of study medication, they will be withdrawn from the study and standard of care treatment will be initiated.

Due to the pragmatic nature of this study and pseudoblinded trial design, at the time of randomization the study subject and corresponding site PIs will be blinded (unaware of specific treatment arm to which the patient is assigned). The study pharmacists as well as data abstractors and designated randomization personnel (i.e., research coordinators and/or research nurses performing the randomization process) will be unblinded.

After randomization and prior to administration of the study medication, blood will be collected to assess laboratory values and relevant medications will be recorded as per - Table 3.

\section{Primary and Secondary Efficacy Outcomes}

The primary efficacy outcome is the composite of VTE (including symptomatic DVT of the upper or lower extremity, 
Table 3 Laboratory values and relevant medications recorded at randomization and day $10+4$ or discharge follow-up

\begin{tabular}{|c|c|c|}
\hline & Randomization & $\begin{array}{l}\text { Day } 10+4 \text { or } \\
\text { discharge } \\
\text { follow-up }\end{array}$ \\
\hline \multicolumn{3}{|l|}{ Laboratory values } \\
\hline $\begin{array}{l}\text { Hemoglobin/ } \\
\text { hematocrit }\end{array}$ & $x$ & $x$ \\
\hline PLT count & $x$ & $x$ \\
\hline $\mathrm{PT} / \mathrm{INR}$ & $x$ & $x$ \\
\hline Serum creatinine & $x$ & $x$ \\
\hline D-Dimer & $x$ & $x$ \\
\hline C-Reactive protein & $x$ & $x$ \\
\hline Fibrinogen & $x$ & $x$ \\
\hline Troponin T/I & $x$ & $x$ \\
\hline $\begin{array}{l}\text { Protein C/protein S } \\
\text { antigen }\end{array}$ & $x$ & \\
\hline Antithrombin activity & $x$ & \\
\hline Quick SOFA score & $x$ & $x$ \\
\hline ISTH SIC score & $x$ & $x$ \\
\hline IMPROVE VTE score & $x$ & $x$ \\
\hline \multicolumn{3}{|l|}{ Medications } \\
\hline $\begin{array}{l}\text { Antiplatelet agents } \\
\text { (aspirin, clopidogrel, } \\
\text { ticagrelor, prasugrel, } \\
\text { vorapaxar, cangrelor) }\end{array}$ & $x$ & $x$ \\
\hline tPA & $x$ & $x$ \\
\hline Steroids & $x$ & $x$ \\
\hline Chloroquine & $x$ & $x$ \\
\hline Hydroxychloroquine & $x$ & $x$ \\
\hline Hormonal therapy & $x$ & $x$ \\
\hline Famotidine & $x$ & $x$ \\
\hline $\begin{array}{l}\text { Immunosuppressant/ } \\
\text { immunomodulatory } \\
\text { agents }\end{array}$ & $x$ & $x$ \\
\hline Antivirals & $x$ & $x$ \\
\hline $\begin{array}{l}\text { Nonsteroidal } \\
\text { anti-inflammatory } \\
\text { drugs }\end{array}$ & $x$ & $x$ \\
\hline
\end{tabular}

Abbreviations: INR, international normalized ratio; ISTH, International Society on Thrombosis and Haemostasis; PLT, platelet; PT, prothrombin time; SIC, sepsis-induced coagulopathy; SOFA, Sequential Organ Failure Assessment; tPA, tissue plasminogen activator; VTE, venous thromboembolism.

asymptomatic proximal DVT of the lower extremity, nonfatal PE), ATE (including MI, stroke, systemic embolism, major adverse limb event, intracardiac thrombus), and ACM at $30 \pm 2$ days after enrollment as per - Table 1 .

The secondary efficacy outcomes include (1) the composite of VTE, ATE, and ACM at day $10+4$ after hospital admission, (2) progression to ARDS, (3) the need for intubation, (4) rehospitalization, (5) new-onset AF, (6) AKI, and (7) nonfatal cardiac arrest at $30 \pm 2$ days after enrollment as per - Table 1 .

\section{Safety Outcomes}

\section{Principal Safety Outcome}

The principal safety outcome is major bleeding at $30 \pm 2$ days after enrollment using validated ISTH bleeding criteria as per - Table 1. Major bleeding is defined as overt bleeding associated with a decrease in hemoglobin of $2 \mathrm{~g} / \mathrm{dL}$ or more within 24 hours, or needing a transfusion of 2 or more units of packed red blood cells, or critical-site bleeding (e.g., intracranial, intraspinal, intraocular, pericardial, intra-articular, intramuscular with compartment syndrome, retroperitoneal), fatal bleeding or bleeding that necessitates surgical intervention.

\section{Serious Adverse Events}

Serious adverse events of special interest will include hypersensitivity reactions, including Stevens-Johnson syndrome, evidence of hepatic toxicity with transaminase elevations greater than six times the ULN, heparin-induced thrombocytopenia using standardized definitions, ${ }^{24}$ and bone marrow toxicity.

\section{Assessment of Outcomes and Follow-Up}

All patients, including those who discontinued study medication, will be followed during their hospitalization and up to day 30 ( \pm 2 days) after study enrollment.

The day $10+4$ visit will occur during hospitalization or at the time of hospital discharge (if this occurs sooner than day 10) and will include a mandatory screening Doppler lower extremity CUS and blood collection to assess laboratory values, as well as an updated list of relevant medications (-Table $\mathbf{3}$ ). Doppler CUS is performed because there is consistent evidence that asymptomatic proximal DVT correlates with ACM in hospitalized medically ill patients, including those with pneumonia or sepsis. ${ }^{25}$ Doppler CUS also is recommended for objective diagnosis in symptomatic patients with suspected upper or lower extremity DVT. Where resource constraints or local institutional policies preclude use of full 5-point Doppler CUS of the lower extremities, point-of-care ultrasound using two-point compression can be substituted. There will be an assessment of all primary efficacy, principal safety, and secondary outcomes during this visit.

The day $30 \pm 2$ visit after enrollment will include an assessment of all primary efficacy, principal safety, and secondary outcomes via a face-to-face or telephone visit.

\section{Data Collection, Sample Size, and Statistical Analysis} Study data obtained during the course of the clinical study will be recorded onsite on paper case report forms (CRFs) and then transferred to REDCap by trained study personnel. The accuracy, completeness, and timeliness of all study procedures will be monitored by the Data Coordinating Center, the EC, and the trial co-PIs, all of which will work together to ensure trial integrity. Copies of paper CRFs will be retained as part of the study record and available for inspection by regulatory authorities. The electronic systems used for data management employ an audit trail that will identify any changes made to study records. 
The effect of therapeutic anticoagulation will be measured by the absolute risk reduction (ARR) defined as the difference in the risk of the primary efficacy endpoint difference between the two treatment arms (arm 0 minus arm 1). The study sample size is determined by an expected $40 \%$ relative risk reduction in the primary efficacy endpoint from $42 \%$ in the prophylactic/ intermediate $\operatorname{arm}(\operatorname{arm} 1)$ to $25.2 \%$ in the treatment $\operatorname{arm}(\operatorname{arm} 0)$ based on earlier reports in sick, hospitalized COVID-19 patients. ${ }^{10}$ Under these assumptions a total of 246 patients (123 in each arm) are needed to provide $80 \%$ power with a twosided significance level of 0.05 . Assuming a 20\% drop-out rate, 308 patients will be recruited and randomized.

The intent-to-treat (ITT) population will consist of all subjects who were randomized. The safety (SAF) and modified ITT (mITT) populations will consist of all randomized patients who received at least one dose of study drug. Reporting of the SAF population will be done according to the majority treatment received (as treated). The Per Protocol (PP) Population will consist of all patients who received at least $80 \%$ of planned therapy and did not have any major protocol deviations. Planned therapy will be calculated as the duration in days that the subject received study treatment according to the randomization arm divided by the duration of hospitalization after randomization, in days.

Major protocol deviations can be assessed from the database and will include those patients who did not meet inclusion criteria or met exclusion criteria, permanently discontinued assigned study medication after randomization not due to an outcome event, and did not undergo the day $10+4$ or discharge screening Doppler lower extremity CUS.

The primary analysis will be done in the PP and mITT populations. The criteria for establishing a significant difference are chosen according to an O'Brien-Fleming design ${ }^{26}$ that includes one interim analysis with early stopping for efficacy of therapeutic-dose anticoagulation compared with the prophylactic/intermediate dose. Under the design assumptions, with 246 patients treatment-dose LMWH will be deemed to be superior to prophylactic/intermediate-dose UFH or LMWH treatment (with two-sided $\alpha$ less than 0.05) if upon trial completion there are 15 or more primary outcome events in the prophylactic/intermediate anticoagulation-dose group than there are in the therapeutic LMWH-dose group, which with 123 patients per group corresponds to an ARR greater than $12.2 \%$.

An interim analysis for efficacy will be conducted after the primary outcome status is observed on approximately 123 randomized patients ( $\sim 50 \%$ of the ITT population). As at the final analysis, the O'Brien-Fleming decision criteria at the interim analysis are the same as at the final analysis, that is, therapeutic anticoagulation will be deemed to be superior to prophylactic/intermediate anticoagulation if there are 15 or more primary outcome events in the prophylactic/intermediate anticoagulation-dose group than there are in the therapeutic LMWH dose group, which with 61 patients per arm corresponds to an ARR greater than $25.6 \%$.

Every effort will be made to minimize the amount of missing data. Due to the time-sensitive nature, the focus will be on three outcome variables: day $10+4$ primary efficacy and principal safety outcomes or discharge Doppler screening lower extremity CUS and day $30 \pm 2$ primary efficacy and principal safety outcomes. There should be no missing data on the most important component of the primary efficacy endpoint, ACM.

\section{DSMB and EC Activities}

An independent external DSMB will actively monitor interim data to review the ongoing safety of patients and will make recommendations about early study closure or changes to the protocol. The DSMB members will include three voting members, two physicians with relevant medical specialty training and experienced in clinical trials research and one clinical trial statistician. An external DSMB support team will prepare unblinded safety reports and the interim analysis for the DSMB. Adjudication of primary and secondary efficacy and principal safety outcomes will be done locally, with periodic quality assessments by the study co-PIs and EC.

All DSMB members will be free of both substantial intellectual and financial conflicts of interests. The DSMB chair reviews subject safety results every 2 weeks by group assignment, judges whether the overall safety of the project remains acceptable, has ongoing access to unblinded information, and makes recommendations after discussion with the DSMB committee. Upon review of the interim analysis results, the DSMB committee makes recommendations about early study closure or changes to the protocol to the study co-PIs and EC, who have the responsibility to accept, reject, or to modify DSMB recommendations. The DSMB meeting frequency will be at $\sim 25, \sim 50, \sim 75$, and $\sim 100 \%$ enrollment. Furthermore, the detailed operation of the DSMB is governed by a charter describing further details such as frequency of meeting, procedures (including but not limited to periodic safety monitoring), and requirements for reporting.

The EC consists of one study PI as Chair and other study investigators, as well as up to four external members with expertise in antithrombotic trials. This EC will assist the study Chair in managing quality oversight of trial-related activities during the conduct of the clinical trial.

\section{Discussion}

The HEP-COVID trial (NCT04401293) is a phase 3 multicenter, pragmatic, prospective, randomized, pseudo-blinded, active control trial that was designed to answer an important question in the COVID-19 pandemic, namely whether there is improved efficacy and acceptable safety of therapeutic-dose LMWH versus prophylactic-/intermediate-dose LMWH/UFH in reducing major thromboembolism or mortality in high-risk hospitalized COVID-19 patients. The trial is randomizing patients into two strata at the time of randomization: whether they require ICU level of care or not. Lastly, the pragmatic design of the trial and accelerated study startup timeframe is meant to maximize patient enrollment and efficiency of study-specific procedures during the time of the COVID-19 pandemic in a hospitalized setting.

There are consistent data that the risk of both VTE and ATE is elevated in sick hospitalized COVID-19 patients, with a 
significant proportion of mortality presumed to be secondary to subclinical thrombosis. ${ }^{2-4,10-12}$ Very elevated thrombotic event rates of $31 \%$ or more and ACM rates of $13 \%$ or more have been described in hospitalized COVID-19 patients requiring ICU level of care. ${ }^{2,27}$ In addition, D-dimer levels greater than four or six times the ULN or elevated SIC scores have been recognized as independent predictors of thrombotic events and poor outcomes. ${ }^{14}$ Consistent with these findings, multivariate analysis using data from a cohort of 9,407 patients with COVID-19 hospitalized at Northwell Health revealed that compared with lower levels, D-dimer levels four to six times the ULN were associated with a 2.1-fold higher risk of VTE or mortality. ${ }^{28}$ Accordingly, this trial uses inclusion criteria anchored on D-dimers at least four times the ULN or an SIC score of 4 or more to ensure enrollment of a high-risk population. The success of this approach is evidenced by the current blinded pooled primary efficacy event rate of $27 \%$. Lastly, a key design feature of the HEP-COVID trial is the inclusion of a screening Doppler lower extremity CUS to capture asymptomatic proximal DVT as a key component of the primary efficacy outcome. There are now consistent data from three large-scale thromboprophylaxis trials that asymptomatic proximal DVT captured by screening ultrasonography is a relevant endpoint in thromboprophylaxis trials and is significantly associated with $\mathrm{ACM}$, with the most recent analysis showing a hazard ratio (HR) of $2.31^{25}$.

The key trial hypothesis is that treatment-dose anticoagulation with LMWH versus standard of care prophylactic-tointermediate doses of $\mathrm{LMWH} / \mathrm{UFH}$ will be more efficacious and have an acceptable safety profile as thromboprophylaxis of major thromboembolic events and ACM in sick, hospitalized COVID-19 patients. A unique feature of COVID-19 coagulopathy is the observation that "breakthrough thrombosis" occurs despite standard thromboprophylaxis, especially in critically ill patients. ${ }^{29}$ Despite prophylactic-dose anticoagulation, a thrombotic complication rate of $16.7 \%$ in COVID-19 patients with ARDS was seen in one study, whereas another study revealed that despite standard thromboprophylaxis in the ICU, VTE rates of $27 \%$, ATE rates of $3.7 \%$, and ACM of $13 \%$ were noted. $^{2,13}$ Preliminary data suggested that exposure to escalated/treatment-dose anticoagulation over standard-ofcare dosing for thromboprophylaxis at index hospitalization for COVID-19 conferred a mortality advantage at 28 days ( 32.8 vs. $52.4 \%, p=0.017$ ) and that therapeutic-dose anticoagulation reduced hospital-based thrombotic complications (HR: 0.29, 95\% confidence interval: 0.091-0.92) ${ }^{10,15}$ Moreover, a recent small randomized trial of therapeutic versus prophylactic anticoagulation in patients with severe COVID-19 revealed that therapeutic anticoagulation improved ventilator-free days. ${ }^{30}$ However, more recent studies have found either no benefit between prophylactic and therapeutic anticoagulation or have found that in-hospital mortality was 2.3 times greater with preemptive treatment-dose anticoagulation from the time of hospital admission. ${ }^{15,31}$ Lastly, whether the mechanisms of thrombosis in COVID-19 are due primarily to in situ immunothrombosis or classic thromboembolic macrovessel disease continues to be a matter of debate, with the former theoretically less susceptible and more resistant to heparin-based strategies for management, even with escalating or treatment doses..$^{32}$ In short, there continues to remain true clinical equipoise for both the efficacy and safety of using treatment-dose anticoagulation for primary thromboprophylaxis in this high-risk hospitalized COVID-19 population.

Limitations of the HEP-COVID trial include the pseudoblinded trial design, although unlike a complete open-label design, we have attempted to mitigate any impact on outcomes by blinding both study investigators and participants. We acknowledge that blinding the receipt of some of the study medications (i.e., IV UFH using a nomogram) may be difficult to do. In addition, the pragmatic design of the trial allows flexibility of investigators to utilize local thromboprophylactic regimens and doses (including both UFH and LMWH from prophylactic-to-intermediate doses) for the standard-of-care thromboprophylactic arm. Although this may enhance the external generalizability of trial results, this also introduces heterogeneity of regimens and doses used to define the standard-of-care thromboprophylactic arm. Another potential limitation is the use of local adjudication to define primary and key secondary as well as principal safety outcomes, although we have attempted to mitigate any reporting inconsistencies by utilizing standardized definitions and performing periodic quality assessments. Lastly, we acknowledge the potential that the trial may be underpowered to answer the clinical question based on our trial's hypothesis of a $42 \%$ event rate of major thromboembolism and ACM in the control group, although to date our pooled primary efficacy event rate of $27 \%$ suggests that we have been successful in enrolling a high-risk subgroup of hospitalized COVID-19 patients.

The HEP-COVID trial is among 20 high-quality global trials (representing a total of 12,568 subjects), including the large multiplatform ATTACC, REMAP-CAP, and ACTIVE-IV trials that at the present time are assessing efficacy and safety of escalated or treatment-dose anticoagulant regimens versus standard-ofcare anticoagulant regimens to reduce COVID-19 coagulopathy in hospitalized patients ( - Table 4 ).$^{33}$ Some of these trials only include critically ill hospitalized COVID-19 patients while others include both ICU and medical-ward patients. ${ }^{33}$ The HEP-COVID trial design where subjects are stratified at the time of randomization to ICU versus non-ICU level of care is designed to potentially stop the trial early in either ICU versus non-ICU populations separately if there is evidence of overwhelming efficacy or futility using prespecified stopping criteria during the interim analysis. At the time of this writing, trial investigators of the multiplatform ATTACC, REMAP-CAP, and ACTIVE-IV trials have paused trial enrollment in the ICU population due to futility concerns as well as potential for harm. ${ }^{34}$ Due to the urgent nature of the clinical question and magnitude of the current COVID-19 pandemic, many investigator groups including ours that are assessing different dosing regimens of anticoagulant interventions in hospitalized COVID-19 patients recognize the need for collaboration to complete trials as soon as possible, pool relevant data when feasible, and disseminate effective interventions as rapidly as possible. Toward this goal, there is a collaborative effort led by the World Health Organization and supported by the INVENT-VTE network (www.INVENT-VTE.com) to conduct a 


\begin{tabular}{|c|c|c|c|c|c|c|c|c|c|c|c|}
\hline 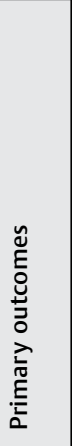 & 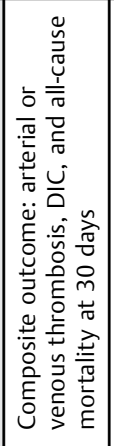 & 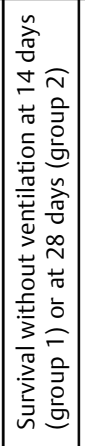 & 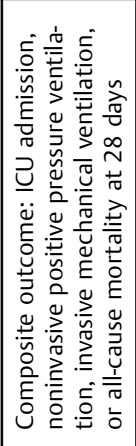 & 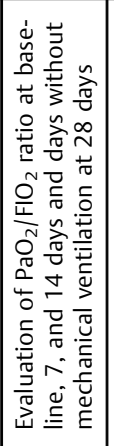 & 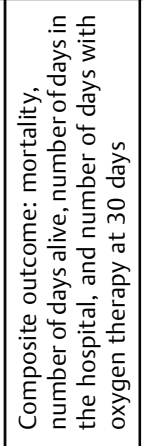 & 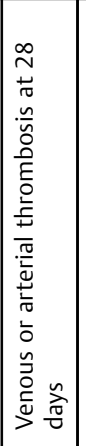 & 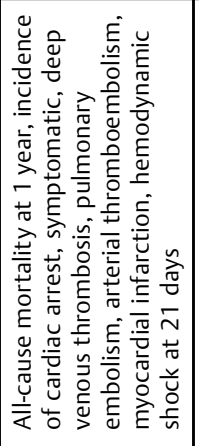 & 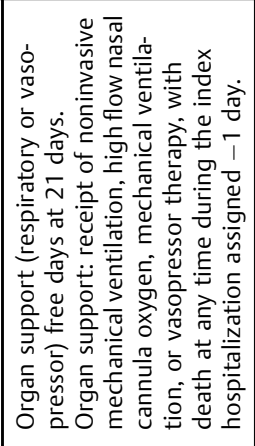 & 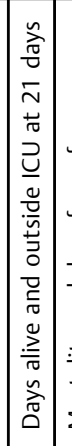 & 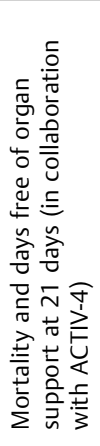 & 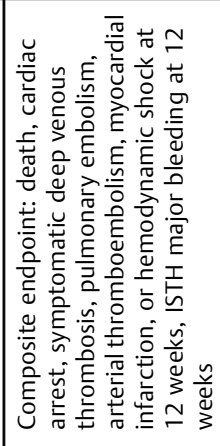 \\
\hline 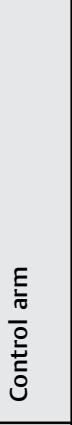 & 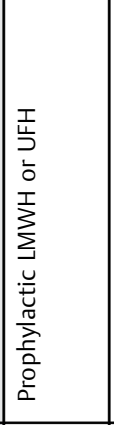 & 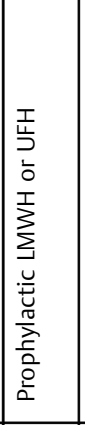 & 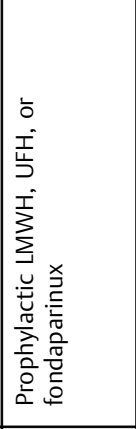 & 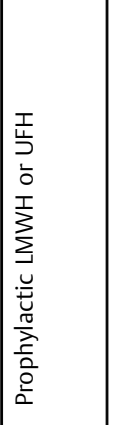 & 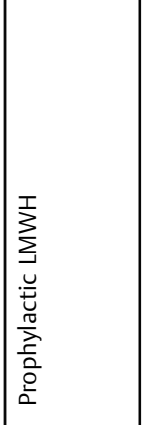 & 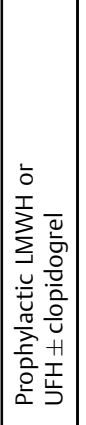 & 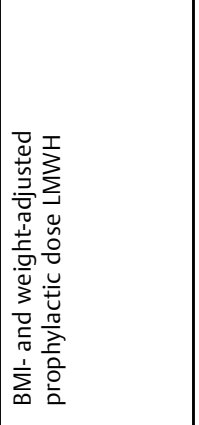 & 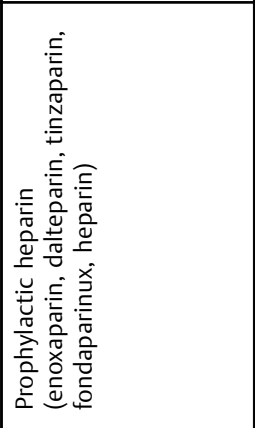 & 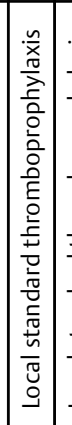 & 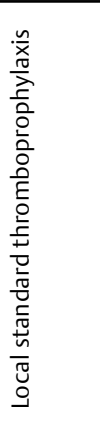 & 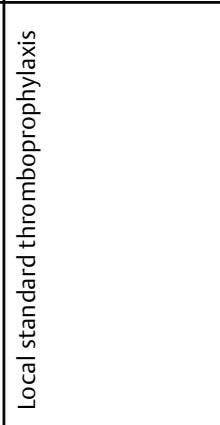 \\
\hline 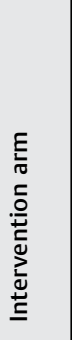 & 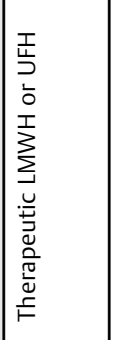 & 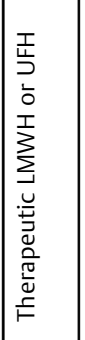 & 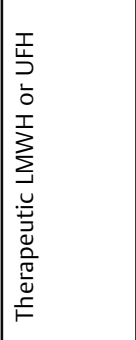 & 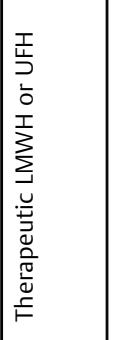 & 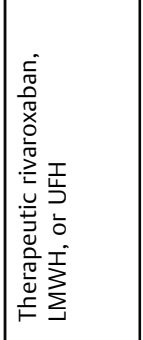 & 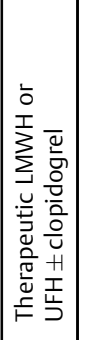 & 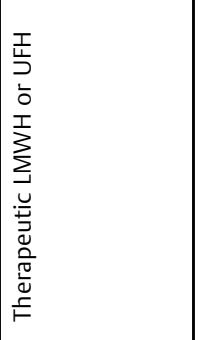 & 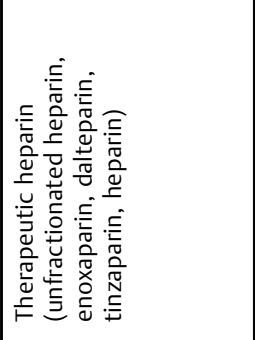 & 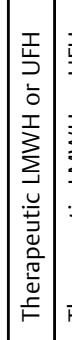 & 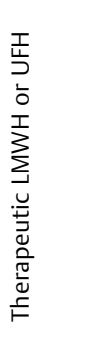 & 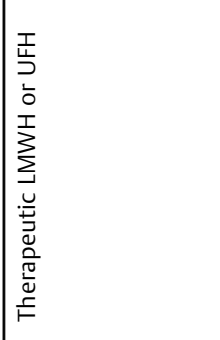 \\
\hline 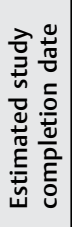 & 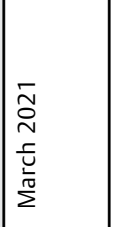 & 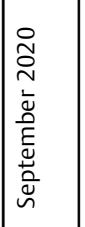 & 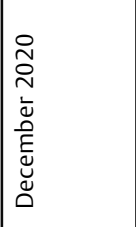 & 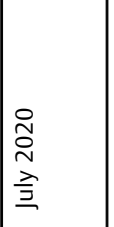 & 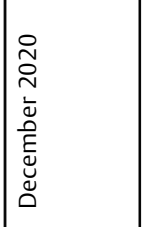 & $\mid \begin{array}{l}\overline{\widetilde{\Omega}} \\
\sim \\
\overline{\tilde{N}} \\
\bar{\Sigma}\end{array}$ & 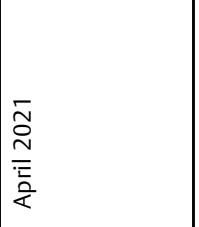 & 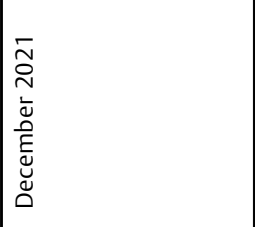 & 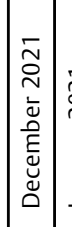 & 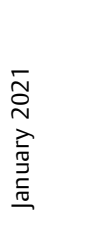 & 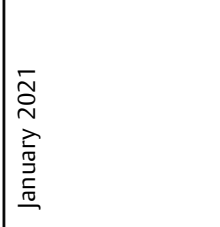 \\
\hline 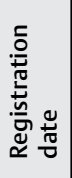 & 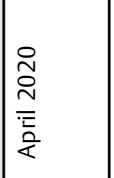 & 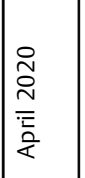 & 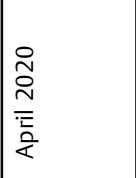 & 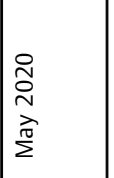 & 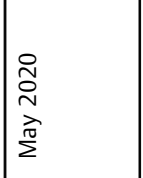 & 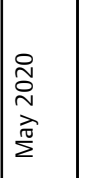 & 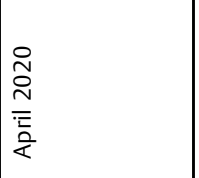 & 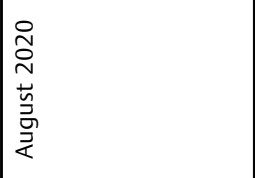 & 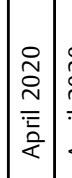 & 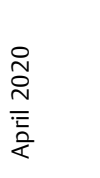 & $\begin{array}{l}\stackrel{2}{\widetilde{N}} \\
\stackrel{N}{1} \\
\stackrel{\pi}{\pi} \\
\stackrel{\pi}{\Sigma}\end{array}$ \\
\hline 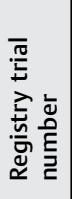 & 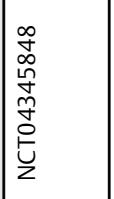 & 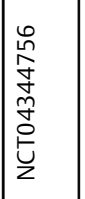 & 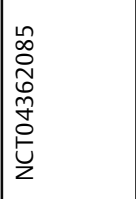 & 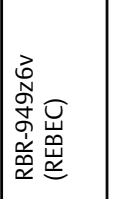 & 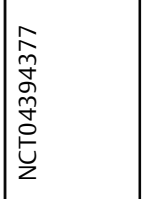 & 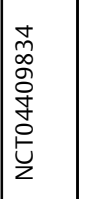 & 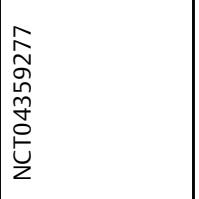 & 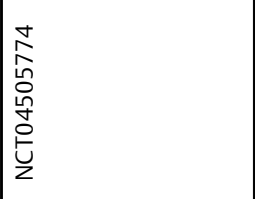 & 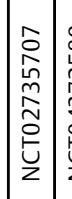 & 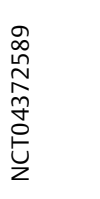 & 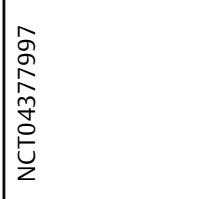 \\
\hline 辛 & 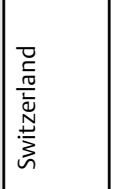 & 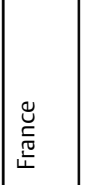 & 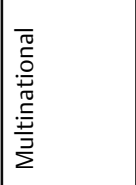 & 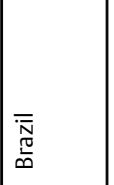 & \begin{tabular}{|l}
$\overline{\bar{N}}$ \\
$\underline{\underline{D}}$ \\
\end{tabular} & 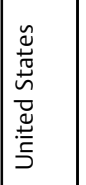 & 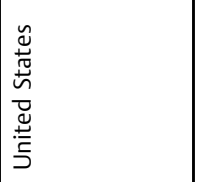 & 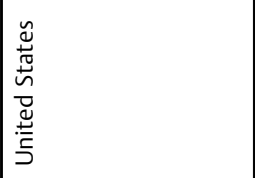 & 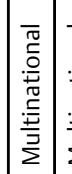 & 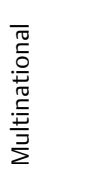 & 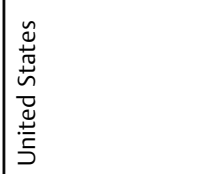 \\
\hline 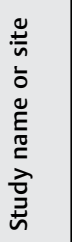 & 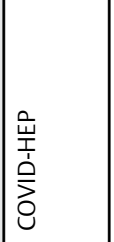 & 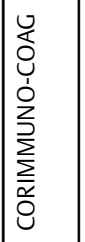 & \begin{tabular}{|l}
0 \\
$\vdots$ \\
0 \\
0 \\
0 \\
$\partial$ \\
0 \\
0 \\
0 \\
$\frac{a}{\alpha}$ \\
$\Sigma$
\end{tabular} & 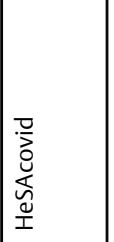 & 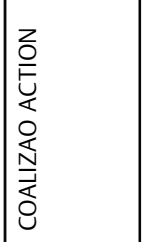 & 号 & 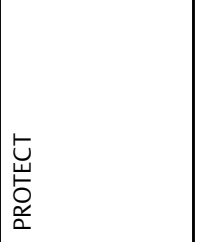 & 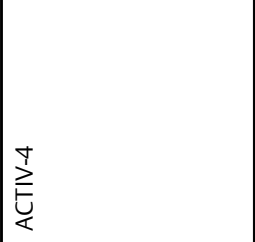 & 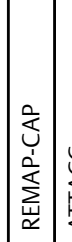 & 荙 & 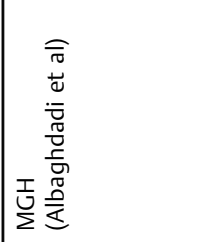 \\
\hline
\end{tabular}




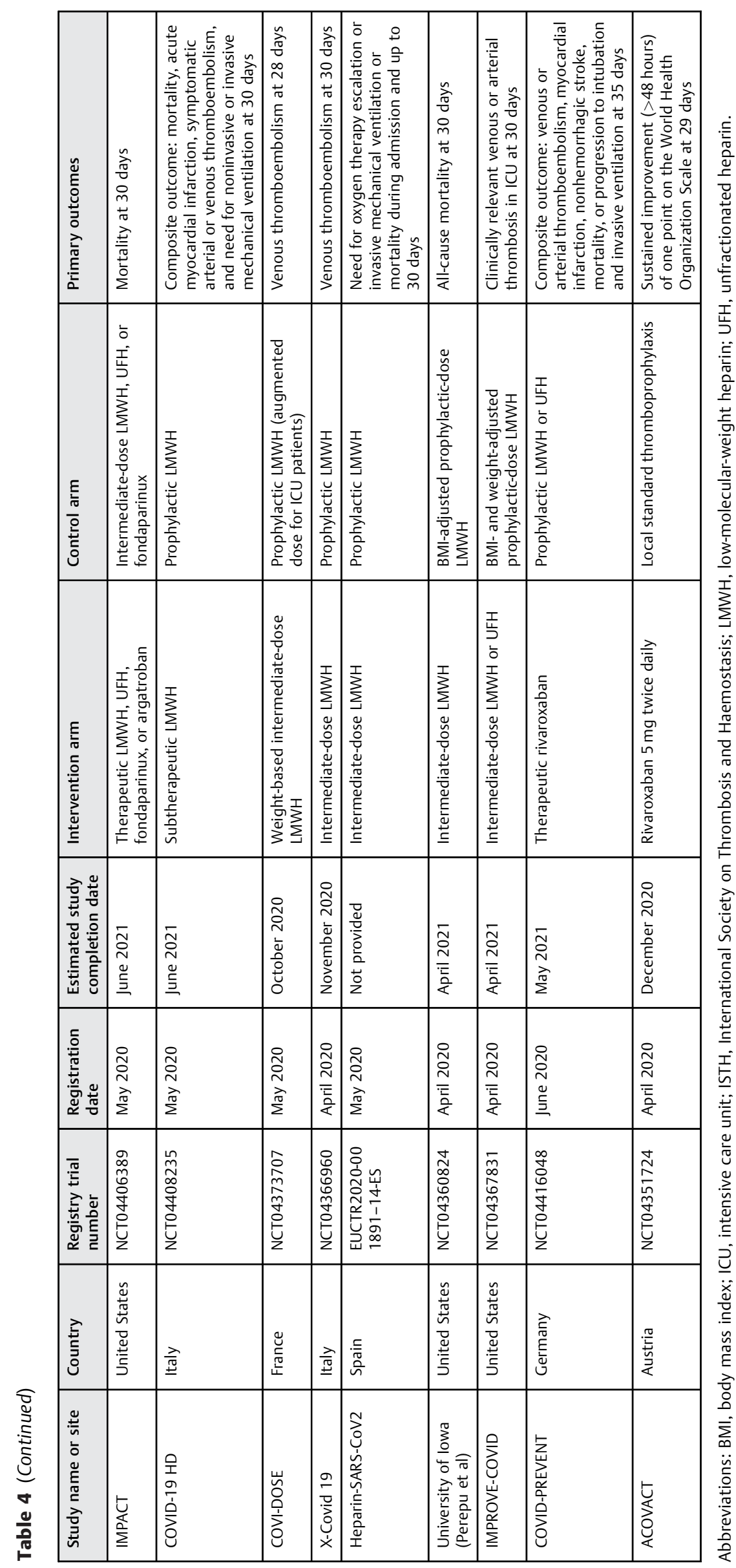

Thrombosis and Haemostasis Vol. $121 \quad$ No. 12/2021 $\quad$ @ 2021. Thieme. All rights reserved. 
prospective meta-analysis to combine key study populations and relevant outcomes from the previously mentioned anticoagulant intervention trials in hospitalized COVID-19 patients. Thus high quality and definitive conclusions from the pooled results of these trials may result in their rapid dissemination to the global clinical community and inform clinical practice antithrombotic guidelines on the topic.

In conclusion, the HEP-COVID trial is a high-quality multicenter randomized trial that is pseudo-blinded and aims to answer a key clinical question, namely, is there net clinical benefit for using treatment-dose LMWH versus prophylactic- or intermediate-dose LMWH/UFH for thromboprophylaxis in high-risk hospitalized COVID-19 patients. It has unique design features and is pragmatic in design. The results of this trial, coupled with other high-quality trials of anticoagulant interventions in this population, has the potential to inform best clinical practice in managing the coagulopathy and potentially reducing the ensuing morbidity and mortality seen in hospitalized COVID-19 patients.

\section{Conflict of Interest}

M.G.: research grant from Janssen; M.C.: speakers bureau Sanofi; A.T.: educational grant: Janssen, research grant: Janssen, Bristol Meyers Squibb, Idorsia, consultant: Recovery Force, J.M.K.: consultant for Bayer Healthcare, Jansen, the ATLAS Group, AMAG pharmaceuticals, Agile Therapeutics for advisory and steering committee activities. Data and safety monitoring committees for Cystic Fibrosis Foundation Therapeutics, Novo Nordisk, Pfizer, Genentech, and BioMarin pharmaceuticals. M.B.: research grant support to CPC Clinical Research from Amgen, AstraZeneca, Bayer, Janssen, Merck, NovoNordisk; J.L.H.: consultancies with the ATLAS Group, Bayer Healthcare, Boehringer Ingelheim, and Population Health Research Institute; J.I.W.: consultant for Anthos, ATLAS Group, Bayer, Boehringer Ingelheim, Bristol Myers Squibb, Daiichi Sankyo, Ionis, Janssen, Johnson and Johnson, Merck, Pfizer, PhaseBio, and Servier, institutional research grants from Bayer and Boehringer Ingelheim; A.C.S.: Janssen, Boehringer Ingelheim, Bayer, BMS, Portola, ATLAS Group: consultancy; Janssen, Boehringer Ingelheim: research funding. D.G., W.D., J.W., S.K., G. S., J.M.L., A.M., E.G., P.A.L., K.C., M.L., C.P.S., H.R., K.O., W.R.H., R.A.D., V.E.A.: no conflict of interest.

\section{Acknowledgments}

The HEP-COVID trial is dedicated to the memory of Dr. Will Hiatt who as part of the trial's Executive Committee and President of CPC Clinical Research was instrumental in initiation of trial activities as well as serving as an inspiration to trial investigators. We would also like to acknowledge Drs. Jim Douketis and Sam Schulman, who as Chair and Member of the trial's DSMB, respectively, have worked tirelessly in their activities. We would finally like to acknowledge all of the local principal investigators, clinical research coordinators (including Damian Inlall who assisted in study initiation activities), and study personnel who have contributed greatly to the HEPCOVID trial. This trial is partly funded from the Broxmeyer
Fellowship in Clinical Thrombosis, as well as by generous donors in our Northwell Health community.

\section{References}

1 Novel Coronavirus. (2019-nCoV) situation reports [Internet] [cited 2020 Dec 28]. Accessed April 18, 2021 at: https://www.who.int/ emergencies/diseases/novel-coronavirus-2019/situation-reports

2 Klok FA, Kruip MJHA, van der Meer NJM, et al. Confirmation of the high cumulative incidence of thrombotic complications in critically ill ICU patients with COVID-19: An updated analysis. Thromb Res 2020;191:148-150

3 Hanif A, Khan S, Mantri N, et al. Thrombotic complications and anticoagulation in COVID-19 pneumonia: a New York City hospital experience. Ann Hematol 2020;99(10):2323-2328

4 Bilaloglu S, Aphinyanaphongs Y, Jones S, Iturrate E, Hochman J, Berger JS. Thrombosis in hospitalized patients with COVID-19 in a New York City health system. JAMA 2020;324(08):799-801

5 Spyropoulos AC, Weitz JI. Hospitalized COVID-19 patients and venous thromboembolism: a perfect storm. Circulation 2020;142 (02):129-132

6 Bikdeli B, Madhavan MV, Gupta A, et al; Global COVID-19 Thrombosis Collaborative Group. Pharmacological agents targeting thromboinflammation in COVID-19: review and implications for future research. Thromb Haemost 2020;120(07):1004-1024

7 Bikdeli B, Madhavan MV, Jimenez D, et al; Global COVID-19 Thrombosis Collaborative Group, Endorsed by the ISTH, NATF, ESVM, and the IUA, Supported by the ESC Working Group on Pulmonary Circulation and Right Ventricular Function. COVID-19 and thrombotic or thromboembolic disease: implications for prevention, antithrombotic therapy, and follow-up: JACC stateof-the-art review. J Am Coll Cardiol 2020;75(23):2950-2973

8 Manne BK, Denorme F, Middleton EA, et al. Platelet gene expression and function in patients with COVID-19. Blood 2020;136 (11):1317-1329

9 Giannis D, Ziogas IA, Gianni P. Coagulation disorders in coronavirus infected patients: COVID-19, SARS-CoV-1, MERS-CoV and lessons from the past. J Clin Virol 2020;127:104362

10 Tang N, Bai H, Chen X, Gong J, Li D, Sun Z. Anticoagulant treatment is associated with decreased mortality in severe coronavirus disease 2019 patients with coagulopathy. J Thromb Haemost 2020;18(05):1094-1099

11 Wichmann D, Sperhake J-P, Lütgehetmann M, et al. Autopsy findings and venous thromboembolism in patients with COVID-19: a prospective cohort study. Ann Intern Med 2020;173(04):268-277

12 Lax SF, Skok K, Zechner P, et al. Pulmonary arterial thrombosis in COVID-19 with fatal outcome: results from a prospective, singlecenter, clinicopathologic case series. Ann Intern Med 2020;173 (05):350-361

13 Helms J, Tacquard C, Severac F, et al; CRICS TRIGGERSEP Group (Clinical Research in Intensive Care and Sepsis Trial Group for Global Evaluation and Research in Sepsis) High risk of thrombosis in patients with severe SARS-CoV-2 infection: a multicenter prospective cohort study. Intensive Care Med 2020;46(06):1089-1098

14 Tang N, Li D, Wang X, Sun Z. Abnormal coagulation parameters are associated with poor prognosis in patients with novel coronavirus pneumonia. J Thromb Haemost 2020;18(04):844-847

15 Paranjpe I, Fuster V, Lala A, et al. Association of treatment dose anticoagulation with in-hospital survival among hospitalized patients with COVID-19. J Am Coll Cardiol 2020;76(01):122-124

16 Stessel B, Vanvuchelen C, Bruckers L, et al. Impact of implementation of an individualised thromboprophylaxis protocol in critically ill ICU patients with COVID-19: a longitudinal controlled before-after study. Thromb Res 2020;194:209-215

17 Nadkarni GN, Lala A, Bagiella E, et al. Anticoagulation, bleeding, mortality, and pathology in hospitalized patients with COVID-19. J Am Coll Cardiol 2020;76(16):1815-1826 
18 Spyropoulos AC, Levy JH, Ageno W, et al; Subcommittee on Perioperative, Critical Care Thrombosis, Haemostasis of the Scientific, Standardization Committee of the International Society on Thrombosis and Haemostasis. Scientific and Standardization Committee communication: clinical guidance on the diagnosis, prevention, and treatment of venous thromboembolism in hospitalized patients with COVID-19. J Thromb Haemost 2020;18(08):1859-1865

19 Moores LK, Tritschler T, Brosnahan S, et al. Prevention, diagnosis, and treatment of VTE in patients with coronavirus disease 2019: CHEST guideline and expert panel report. Chest 2020;158(03): 1143-1163

20 Fernández-Capitán C, Barba R, Díaz-Pedroche MDC, et al. Presenting characteristics, treatment patterns, and outcomes among patients with venous thromboembolism during hospitalization for COVID-19. Semin Thromb Hemost 2021;47(04):351-361

21 Merli G, SpiroTE, Olsson CG, et al; Enoxaparin Clinical Trial Group. Subcutaneous enoxaparin once or twice daily compared with intravenous unfractionated heparin for treatment of venous thromboembolic disease. Ann Intern Med 2001;134(03):191-202

22 Gargiulo G, Carrara G, Frigoli E, et al. Bivalirudin or heparin in patients undergoing invasive management of acute coronary syndromes. J Am Coll Cardiol 2018;71(11):1231-1242

23 Cohoon KP, Mahé G, Tafur AJ, Spyropoulos AC. Emergence of institutional antithrombotic protocols for coronavirus 2019. Res Pract Thromb Haemost 2020;4(04):510-517

24 Cuker A, Arepally GM, Chong BH, et al. American Society of Hematology 2018 guidelines for management of venous thromboembolism: heparin-induced thrombocytopenia. Blood Adv 2018;2(22):3360-3392

25 Raskob GE, Spyropoulos AC, Cohen AT, et al. Association between asymptomatic proximal deep vein thrombosis and mortality in acutely ill medical patients. J Am Heart Assoc 2021;10(05):e019459

26 Emerson SS, Fleming TR. Symmetric group sequential test designs. Biometrics 1989;45(03):905-923
27 Poissy J, Goutay J, Caplan M, et al; Lille ICU Haemostasis COVID-19 Group. Pulmonary embolism in patients with COVID-19: awareness of an increased prevalence. Circulation 2020;142(02):184-186

28 Cohen SL, Gianos E, Barish MA, et al;Northwell Health COVID-19 Research Consortium. Prevalence and predictors of venous thromboembolism or mortality in hospitalized COVID-19 patients. Thromb Haemost 2021;121(08):1043-1053

29 Spyropoulos AC, Weitz JI. Hospitalized COVID-19 patients and venous thromboembolism: a perfect storm. Circulation 2020;142 (02):129-132

30 Lemos ACB, do Espírito Santo DA, Salvetti MC, et al. Therapeutic versus prophylactic anticoagulation for severe COVID-19: a randomized phase II clinical trial (HESACOVID). Thromb Res 2020; 196:359-366

31 Motta JK, Ogunnaike RO, Shah R, et al. Clinical outcomes with the use of prophylactic versus therapeutic anticoagulation in COVID19. medRxiv [Internet] Cold Spring Harbor Laboratory Press; 2020 [cited 2020 Sep 14]. Accessed April 18, 2021 at: https:// www.medrxiv.org/content/10.1101/2020.07.20.20147769v1

32 Loo J, Spittle DA, Newnham M. COVID-19, immunothrombosis and venous thromboembolism: biological mechanisms. Thorax $2021 ; 76: 412-420$

33 Tritschler T, Mathieu M-E, Skeith L, et al; International Network of VENous Thromboembolism Clinical Research Networks INVENTVTE. Anticoagulant interventions in hospitalized patients with COVID-19: a scoping review of randomized controlled trials and call for international collaboration. J Thromb Haemost 2020;18 (11):2958-2967

34 National Institutes of Health. NIH ACTIV Trial of blood thinners pauses enrollment of critically ill COVID-19 patients [Internet]. Natl. Inst. Health NIH. 2020 [cited 2021 Jan 15]. Accessed April 18, 2021 at: https://www.nih.gov/news-events/news-releases/nihactiv-trial-blood-thinners-pauses-enrollment-critically-ill-covid19-patients 